\title{
Erratum to: Immunological and biological changes during ipilimumab treatment and their potential correlation with clinical response and survival in patients with advanced melanoma
}

\author{
Ester Simeone • Giusy Gentilcore • Diana Giannarelli • Antonio M. Grimaldi · Corrado Caracò • \\ Marcello Curvietto $\cdot$ Assunta Esposito $\cdot$ Miriam Paone $\cdot$ Marco Palla $\cdot$ Ernesta Cavalcanti $\cdot$ Fabio Sandomenico $\cdot$ \\ Antonella Petrillo · Gerardo Botti · Franco Fulciniti · Giuseppe Palmieri · Paola Queirolo · Paolo Marchetti · \\ Virginia Ferraresi · Gaetana Rinaldi • Maria Pia Pistillo $\cdot$ Gennaro Ciliberto $•$ Nicola Mozzillo $\cdot$ Paolo A. Ascierto
}

Published online: 25 September 2014

(C) Springer-Verlag Berlin Heidelberg 2014

Erratum to: Cancer Immunol Immunother (2014) 63:675-683

DOI 10.1007/s00262-014-1545-8

Unfortunately, a sentence in the "Efficacy and immunological markers of response" section of the Results was incorrectly published. The correct sentence should read:
The change in absolute numbers of FoxP3/Treg cells showed the same trend as the percentages, i.e. the number of FoxP3/Treg cells in the periphery increased in patients without disease control and decreased in patients with disease control (data not shown).

The online version of the original article can be found under doi:10.1007/s00262-014-1545-8.

E. Simeone · G. Gentilcore · A. M. Grimaldi · C. Caracò ·

M. Curvietto - A. Esposito - M. Paone - M. Palla - E. Cavalcanti ·

F. Sandomenico $\cdot$ A. Petrillo $\cdot$ G. Botti $\cdot$ F. Fulciniti $\cdot$ G. Ciliberto

N. Mozzillo $\cdot$ P. A. Ascierto $(\bowtie)$

Unit of Melanoma, Cancer Immunotherapy and Innovative

Therapy, IRCCS, Istituto Nazionale Tumori Fondazione

"G. Pascale", Via Mariano Semmola, 80131 Naples, Italy

e-mail: paolo.ascierto@gmail.com

D. Giannarelli · V. Ferraresi

Regina Elena National Cancer Institute, Rome, Italy

G. Palmieri

National Research Council, Sassari, Italy

P. Queirolo · M. P. Pistillo

San Martino Hospital, National Institute for Cancer Research,

Genoa, Italy

P. Marchetti

Dermopathic Institute of the Immaculate IDI-IRCCS, Rome, Italy

P. Marchetti

Sant'Andrea Hospital, University Sapienza, Rome, Italy

G. Rinaldi

"Paolo Giaccone" Polyclinic University Hospital, Palermo, Italy 